\title{
11 GENETIC INFLUENCE OF SPECIFIC ANTICOLLAGEN ANTIBODY PRODUCTION IN A MOUSE MODEL OF RHEUMATOID ARTHRITIS
}

Bruno Raposo, Doreen Dobritzsch, ${ }^{2}$ Hüseyin Uysal, ${ }^{1}$ Diana Ekman, ${ }^{1}$ Rikard Holmdahl' ${ }^{1}$ Division of Molecular Structural Biology, Department of Medical Biochemistry and Biophysics, Karolinska Institutet, Stockholm, Sweden; ${ }^{2}$ Division of Medical Inflammation Research, Department of Medical Biochemistry and Biophysics, Karolinska Institutet, Stockholm, Sweden

10.1136/annrheumdis-2011-201236.11

Background and objectives Rheumatoid arthritis (RA) is an autoimmune disease affecting about $1 \%$ of the population. Though of unknown aetiology, it is considered to have a strong genetic influence, resulting in the activation of different immune cells and consequent bone and cartilage destruction of the joints. The production of anticollagen type II (CII) antibodies is a common feature between RA patients and the collagen-induced arthritis (CIA) mouse model. Understanding how distinct anti-CII epitopes are genetically selected could benefit the development of new RA therapies. Here, the authors assess the genetic influence of specific anti-CII antibody production in a mouse model of RA.

Material and methods In this particular study, the authors have used as genetic variance tool a heterogeneous stock (HS) mice cohort of eight inbred mouse strains. Three of the major anti-CII B cell epitopes (C1, U1 and J1) were analysed by ELISA. Sequencing of the genes coding for the M2139 monoclonal antibody (J1-specific $\mathrm{mAb}$ ) was additionally done for all the HS strains.

Results A genome wide study of antibody response during CIA development suggested that specific anti-CII antibodies are regulated by the immunoglobulin heavy chain locus $(\operatorname{Ig} \mathrm{H})$. The haplotype reconstruction analysis showed a preference for the anti-J1 antibody production by certain mouse strains, in detriment of anti-C1 antibodies. The authors thus hypothesised that the selection of particular IgHv genes is responsible for this J1 versus C1 epitope selection. Preliminary sequencing data of the genes coding for the M2139 monoclonal antibody in all the HS strains, supports the initial hypothesis. The difference relies on an amino acid change from a hydrophobic tryptophan to a polar amino acid (eg, threonine) in the CDR1 region. Three-dimensional structure of M2139 Fab with triple helical J1 peptide shows that the tryptophan in the CDR1 region of the heavy chain is contacting with a hydrophobic leucine amino acid in the J1 epitope. This interaction may be essential for 
recognition of the J1 epitope. Replacement of Trp by Thr in this position could abrogate the binding to J1 epitope.

Conclusions CIA development in HS mice allowed for a genome wide study on anti-CII specific responses. The data revealed a genetic association with the production of anti-J1 antibodies. Sequencing of the genes coding for the anti-J1 $\mathrm{mAb}$ reveal an amino acid substitution influencing the polarity within the CDR1 region. Cloning and expression of these mutations and consequent anti-J1 binding properties will further on confirm or reject our hypothesis. 